\title{
The risk for breast cancer is not evidently increased in women with hyperprolactinemia
}

\author{
O. M. Dekkers · J. A. Romijn • A. de Boer • \\ J. P. Vandenbroucke
}

Published online: 12 December 2009

(C) The Author(s) 2009. This article is published with open access at Springerlink.com

\begin{abstract}
The question has been raised whether hyperprolactinemia in humans is associated with an excess risk for breast cancer. We aimed to assess the risk of breast cancer in a previously defined large cohort of patients treated for idiopathic hyperprolactinemia or prolactinomas. Based on the pattern of drug prescriptions we identified 11,314 subjects in the PHARMO network with at least one dispensing of dopamine agonists between 1996 and 2006. Of these, 1,607 subjects were considered to have dopamine agonist-treated hyperprolactinemia based on the prescribing pattern. For the present analysis, we included only women $(n=1,342)$. Patients with breast cancer were identified by hospital discharge codes. Data on breast cancer incidence in the Netherlands were derived from the Dutch cancer registry. Standardized mortality ratio (SMR) was the measure of outcome to assess the association between hyperprolactinemia and breast cancer. The 1,342 patients accounted for a total of 6,576 person years. Eight patients with breast cancer during follow-up were identified. Indirect standardization with incidence proportions from the general Dutch population revealed a 7.47 expected cases. The calculated SMR for breast
\end{abstract}

O. M. Dekkers · J. A. Romijn

Department of Endocrinology and Metabolic Diseases, Leiden University Medical Center, P.O. Box 9600, 2300 RC Leiden,

The Netherlands

O. M. Dekkers ( $($ ) · J. P. Vandenbroucke

Department of Clinical Epidemiology, Leiden University

Medical Center, P.O. Box 9600, 2300 RC Leiden,

The Netherlands

e-mail: o.m.dekkers@lumc.nl

\section{A. de Boer}

Division of Pharmacoepidemiology and Pharmacotherapy,

Utrecht Institute for Pharmaceutical Sciences,

Utrecht University, Utrecht, The Netherlands cancer risk in patients treated hyperprolactinemia was 1.07 (95\% confidence interval 0.50-2.03). In conclusion, there is no clear evidence for increased breast cancer risk in female patients treated for either idiopathic hyperprolactinemia or prolactinomas. The uncertainty about the exact risk that is due to the relatively low number of breast cancer cases, should be overcome by pooling results in a future meta-analysis.

Keywords Hyperprolactinemia - Breast cancer . SMR

\section{Introduction}

Hyperprolactinemia that causes complaints and leads to treatment is caused by a prolactinoma in the majority of cases [1]. In the absence of visualization of an adenoma on MRI, and of secondary causes such as medication, the hyperprolactinemia is referred to as idiopathic [2]. Low dose of a dopamine agonist is the treatment of choice in patients with a prolactinoma or with complaints caused by hyperprolactinemia. Dopamine agonists normalize prolactin levels and decrease tumor size in the majority of cases [3]. However, due to a delay in diagnosis, these patients often have been exposed to increased levels of prolactin for months to years.

Prolactin has proliferative effects on mammary epithelial cells [4]. Moreover, hyperprolactinemia is associated with oncogenesis of the mammary glands in rodents [5]. However, the issue is still unresolved, whether hyperprolactinemia is associated with an excess risk for breast cancer in female subjects $[5,6]$. Nonetheless, case reports have suggested an association between prolactinomas and breast cancer [7, 8]. In follow-up studies higher prolactin levels have been associated with an increased risk for 
breast cancer $[9,10]$. Unfortunately, studies on prolactinoma patients are often underpowered to assess the risk for breast cancer properly. Therefore, the aim of the present analysis was to assess the risk of breast cancer in a previously defined large cohort of patients treated for idiopathic hyperprolactinemia or prolactinomas.

\section{Patients and methods}

\section{Study design and setting}

We identified an open cohort of patients treated with dopamine agonists for hyperprolactinemia using the PHARMO network (www.pharmo.nl). This database includes the demographic details and virtual complete medication histories from community pharmacies for more than 2 million community-dwelling residents of 25 geographic areas in the Netherlands from 1985 to the present. These data are also linked to all hospital admissions of these subjects throughout the Netherlands. All hospital discharge codes are coded according to the International Classification of Diseases, Ninth Revision, Clinical Modification (ICD-9 CM). The ICD-9 CM codes 1,740-1,749 and 2,330 were used to define the diagnosis of breast cancer for the present analysis. Data on breast cancer incidence on the Netherlands were derived from the Dutch cancer registry (www.ikcnet.nl).

According to Dutch law, there is no requirement of informed consent if the patient data are completely anonymous, cannot be traced to individual persons and patients are not contacted for intervention purposes. Therefore, our investigation using the PHARMO network complies with all ethics and privacy regulations in the Netherlands.

\section{Patients}

Based on prescription patterns we identified 11,314 subjects with at least one dispensing of dopamine agonists in the period 1996-2006. Of these subjects, 1,607 subjects were considered to have dopamine agonist-treated hyperprolactinemia based on the prescribing pattern. Details on this cohort have been published previously [11]. For the present analysis on the association between breast cancer risk and hyperprolactinemia we only included women $(n=1,342)$.

\section{Analysis}

\section{Breast cancer}

Hospital discharge codes were used to identify patients with a diagnosis of breast cancer. This is a reliable marker for breast cancer, since breast cancer is almost invariable treated primarily by surgery, requiring hospital admission.
Prior to start of data analysis, it was decided only to count breast cancer cases that were diagnosed after start of the dopamine-agonist treatment for hyperprolactinemia. The reason is that in prolactinomas the diagnosis is often delayed, and the patients may have been exposed to elevated prolactin levels years before the diagnosis [12].

\section{Person-years}

Person-years were calculated by adding the follow-up time of all individual patients with dopamine-agonist treated hyperprolactinemia. For the purpose of the present analysis, person time was split in age bands of 5 years. For all patients the follow-up time was started on the date of the first prescription of a dopamine agonist in the database. End of follow-up time was determined by the date of the last prescription in the database for that particular patient, irrespective whether this was for a dopamine-agonist, or for another reason.

\section{Incidences in the Netherlands}

For the purpose of the analysis the average incidence of the breast cancer in The Netherlands of the calendar years 1996-2006 was taken. The incidences were stratified by 5 years of age.

\section{Standardized mortality ratio}

Standardized mortality ratio (SMR) was the measure of outcome to assess the association between hyperprolactinemia and breast cancer. In a SMR the observed number of breast cancer cases in the cohort was compared to the expected number of cases that would have occurred theoretically if the breast cancer risk in the general population is applied to the calculated person-years from the hyperprolactinemia cohort.

\section{Results}

Patients and person-years

The original database consisted of 11,314 subjects with at least one prescription of dopamine agonists with a total of 37,380 prescriptions of dopamine agonists in the period from January 1, 1996 and December 31, 2006. A total of 1,607 patients with dopamine agonist - treated hyperprolactinemia were identified [11]. For the present analysis only women were included $(n=1,342)$.

Mean age of treated female patients was $36 \pm 11$ years. The initial treatment was bromocriptine in 545 patients (41\%), cabergoline in 556 patients $(41 \%)$ and quinagolide 
in 241 patients $(18 \%)$. Pituitary surgery was performed in 31 patients. The mean initial treatment duration was 16.5 months. In 515 patients, dopamine agonist therapy was restarted.

\section{Breast cancer}

Based on hospital discharge codes, eight patients with breast cancer during follow-up were identified. The youngest patient was 38 years at the time of diagnosis, the oldest was 61 years. Breast cancer was diagnosed from 11 months to 8 years and 5 months after the first prescription of a dopamine agonist.

\section{Standardized mortality ratio}

The 1,342 patients accounted for a total 6,576 person years of follow up. Indirect standardization with incidence proportions from the general Dutch population revealed a total number of expected cases of 7.47. The calculated SMR for breast cancer risk in patients treated hyperprolactinemia was 1.07 (95\% confidence interval 0.50-2.03).

\section{Discussion}

The present analysis was conducted to estimate the risk of breast cancer in a large cohort of patients treated for hyperprolactinemia. Compared to the general Dutch population, there was no clearly increased risk (SMR 1.07). However, because the number of patients with breast cancer was rather low, the confidence intervals of this analysis are rather broad, thereby prohibiting a definitive conclusion on the possible association between breast cancer risk and hyperprolactinemia.

This study has limitations. Although the cohort of included patients of dopamine agonist-treated hyperprolactinemia is relatively large, the number of breast cancer cases during follow-up was rather small $(n=8)$. The low number of breast cancer cases is due to two facts: firstly, a relatively short follow-up period of about 5 years and secondly the relatively young age of patients at diagnosis. Because the confidence interval for a SMR is based on the number of events, i.e. the number of patients who develop breast cancer, and not on the number of patients in the cohort, the confidence intervals were wide. This limitation can only be overcome by including even larger cohorts, or by pooling existing data in a meta-analysis. Secondly, the diagnosis of hyperprolactinemia was based on prescriptions of dopamine agonists in a large pharmaco-epidemiological database. This has been shown to be a reliable approach for the identification of patients idiopathic hyperprolactinemia or prolactinomas [11]. Another limitation of the present analysis is the fact that no prolactin values of individual patients were known. Therefore, analysis according to initial prolactin levels was not possible. Moreover, stratified analysis according to disease duration was not possible. Selection bias is a potential other limitation. The diagnoses in our cohort were based on hospital discharge codes, whereas the diagnoses from the Dutch cancer registry were based on either hospital discharge codes or on tissue diagnosis. Since breast cancer is almost invariable treated primarily by surgery, requiring hospital admission, the selection bias will not be large.

Exposure to overproduction of pituitary hormones can lead to an increased risk for cancer, as has been shown in acromegaly [13].The suggested association between breast cancer and elevated levels of prolactin was based on case reports [7, 8], basic research [5] and epidemiological studies [9, 10]. However, thus far, no association between breast cancer risk and idiopathic hyperprolactinemia/prolactinoma has been shown. This is mainly due to the fact that the majority of presented series with prolactinomas are underpowered to detect a possible association [13].

In the present analysis there was no clear association between breast cancer and patients treated for hyperprolactinemia. The inclusion of both prolactinomas and idiopathic hyperprolactinemia enhances the generalizability of the results [14]. However, the ultimate verdict on the association between prolactinomas and breast cancer would require a larger cohort. Despite this limitation our study is incompatible with a large breast cancer risk, i.e. a relative risk $>2$. Several considerations have to be taken into account when interpreting this result. Firstly, the association was assessed in female patients, whereas some case reports described a male patient with breast cancer [7, 15]. In female patients the levels of prolactin at presentation are clearly lower than in men, mainly because men present more often with larger adenomas [16]. In our database, breast cancer was not diagnosed in any of the male patients. Secondly, all patients in the cohort included for the present analysis were treated with dopamine agonists. This treatment is very effective in normalizing prolactin levels [17]. This means that in the follow-up period after start of the medication prolactin levels can be assumed to be normalized in the vast majority of cases. However, in most patients the diagnosis is often delayed and the disease can therefore be accompanied by long-term overexposure to prolactin [12]. Thirdly, estrogens are known to increase the risk of breast cancer [4]. Elevation of prolactin leads to suppression of the estrogen-production by inhibition of the release of luteinizing hormone and the follicle stimulating hormone [3]. The net effect of prolactin on breast cancer risk is therefore also dependent on the degree of suppression of the estrogen production. Theoretically, an increased risk for breast cancer due to 
hyperprolactinemia can be counterbalanced by the decreased production of estrogens.

In conclusion, this study showed no clear evidence for increased breast cancer risk in female patients treated for ether idiopathic hyperprolactinemia or prolactinomas. The uncertainty about the exact risk that is due to the relatively low number of breast cancer cases, should be overcome by pooling results in a future meta-analysis.

Open Access This article is distributed under the terms of the Creative Commons Attribution Noncommercial License which permits any noncommercial use, distribution, and reproduction in any medium, provided the original author(s) and source are credited.

\section{References}

1. Gillam MP, Molitch ME, Lombardi G, Colao A (2006) Advances in the treatment of prolactinomas. Endocr Rev 27:485-534

2. Schlechte JA (2003) Clinical practice. Prolactinoma. N Engl J Med 349:2035-2041

3. Mancini T, Casanueva FF, Giustina A (2008) Hyperprolactinemia and prolactinomas. Endocrinol Metab Clin North Am 37:67-99 viii

4. Hankinson SE (2005) Endogenous hormones and risk of breast cancer in postmenopausal women. Breast Dis 2005(24):3-15

5. Harvey PW, Everett DJ, Springall CJ (2008) Adverse effects of prolactin in rodents and humans: breast and prostate cancer. J Psychopharmacol 22:20-27

6. Goodman G, Bercovich D (2008) Prolactin does not cause breast cancer and may prevent it or be therapeutic in some conditions. Med Hypotheses 70:244-251

7. Forloni F, Giovilli M, Pecis C, Bortolani E, Preziosi A, Barzaghi ME et al (2001) Pituitary prolactin-secreting macroadenoma combined with bilateral breast cancer in a 45 -year-old male. J Endocrinol Invest 24:454-459
8. Strungs I, Gray RA, Rigby HB, Strutton G (1997) Two case reports of breast carcinoma associated with prolactinoma. Pathology 29:320-323

9. Tworoger SS, Sluss P, Hankinson SE (2006) Association between plasma prolactin concentrations and risk of breast cancer among predominately premenopausal women. Cancer Res 66:2476-2482

10. Tworoger SS, Eliassen AH, Sluss P, Hankinson SE (2007) A prospective study of plasma prolactin concentrations and risk of premenopausal and postmenopausal breast cancer. J Clin Oncol 25:1482-1488

11. Kars M, Souverein PC, Herings RM, Romijn JA, Vandenbroucke JP, de Boer A et al (2009) Estimated age- and sex-specific incidence and prevalence of dopamine agonist-treated hyperprolactinemia. J Clin Endocrinol Metab 94:2729-2734

12. Kars M, van der Klaauw AA, Onstein CS, Pereira AM, Romijn JA (2007) Quality of life is decreased in female patients treated for microprolactinoma. Eur J Endocrinol 157:133-139

13. Popovic V, Damjanovic S, Micic D, Nesovic M, Djurovic M, Petakov M et al (1998) Increased incidence of neoplasia in patients with pituitary adenomas. The pituitary study group. Clin Endocrinol (Oxf) 49:441-445

14. Dekkers OM, Elm EV, Algra A, Romijn JA, Vandenbroucke JP (2009) How to assess the external validity of therapeutic trials: a conceptual approach. Int J Epidemiol April 17 [Epub ahead of print]

15. Volm MD, Talamonti MS, Thangavelu M, Gradishar WK (1997) Pituitary adenoma and bilateral male breast cancer: an unusual association. J Surg Oncol 64:74-78

16. Colao A, Sarno AD, Cappabianca P, Briganti F, Pivonello R, Somma CD et al (2003) Gender differences in the prevalence, clinical features and response to cabergoline in hyperprolactinemia. Eur J Endocrinol 148:325-331

17. Colao A, Di Sarno A, Guerra E, Pivonello R, Cappabianca P, Caranci $F$ et al (2007) Predictors of remission of hyperprolactinaemia after long-term withdrawal of cabergoline therapy. Clin Endocrinol (Oxf) 67:426-433 